PHYSICAL REVIEW D 91, 059901(E) (2015)

\title{
Publisher's Note: Muons in air showers at the Pierre Auger Observatory: Mean number in highly inclined events [Phys. Rev. D 91, 032003 (2015)]
}

\author{
A. Aab et al. \\ (Pierre Auger Collaboration)
}

(Received 24 February 2015; published 9 March 2015)

DOI: 10.1103/PhysRevD.91.059901

PACS numbers: 96.50.sd, 13.85.Tp, 98.70.Sa, 99.10.Fg

This paper was published online on 6 February 2015 with two incorrect names in the author list. "J. J. Matthews" should read as "J. A. J. Matthews," and "A. J. Matthews" should read as "J. Matthews." The author names have been corrected as of 24 February 2015. The author names are correct in the printed version of the journal. 